\title{
Current prescription of prophylactic factor infusions and perceived adherence for children and adolescents with haemophilia: a survey of haemophilia healthcare professionals in the United States
}

\author{
C. D. THORNBURG,, S. CARPENTER, $\dagger$ S. ZAPPA, + J. MUNN $\$$ and C. LEISSINGER \\ *Department of Pediatrics, Duke University Medical Center, Durham, NC; $\dagger$ Department of Pediatrics, Children's Mercy \\ Hospital, Kansas City, MO; Department of Pediatrics; $\$$ Cook Children’s Medical Center, Forth Worth, TX; $\$$ Department of \\ Pediatrics, University of Michigan Medical Center, Ann Arbor, MI; and Department of Medicine, Tulane University, New \\ Orleans, LA, USA
}

Summary. The primary goal of prophylaxis in patients with severe haemophilia is to convert the phenotype from severe to moderate and to prevent the development of chronic arthropathy. Prior studies have demonstrated that prophylaxis decreases episodes of joint bleeds and chronic arthropathy. Effectiveness depends on prescription of prophylaxis and adherence to the prescribed regimen. The aim of this study was to determine if prescription of prophylaxis for children with haemophilia and perceptions of adherence to prophylaxis have changed since publication of the Joint Outcome Study (JOS). A questionnaire was sent, in electronic and written formats, to health professionals who provide care to children with haemophilia at US haemophilia treatment centres (HTCs). The response rate was 56 of 128 (44\%) of the targeted HTCs. There were a few missing data and denominators are provided. All responses agreed with the results of the JOS and 30/55 (55\%) reported the JOS increased their prescription of prophylaxis. Nineteen of $56(34 \%)$ physicians or HTC staff reported that they had not prescribed prophylaxis within the last year due to concerns about adherence, and 19/56 (34\%) reported they had stopped prophylaxis due to concerns about adherence within the last year. Predicted adherence decreased with increasing age. Prescription of prophylaxis appears to be increasing since publication of the JOS. Strategies to improve adherence may increase the likelihood of physician prescription of prophylaxis and make prophylaxis easier to implement for individual patients, thereby improving the clinical outcome of children and adults with haemophilia.

Keywords: haemarthrosis, haemophilia, prophylactic treatment, treatment adherence

\section{Introduction}

Patients with severe haemophilia are at risk for frequent joint haemarthrosis. Chronic arthropathy develops after repeated joint haemarthrosis and is associated with muscle wasting, morning stiffness, limited movement, chronic pain, decreased functional status and poor quality of life; chronic arthropathy is a devastating and costly complication of haemophilia [1]. Prophylactic factor replacement (prophylaxis) is given 1-4 times per week to prevent bleeding; many treaters attempt to

Correspondence: Courtney D. Thornburg, MD, MS, Duke University Medical Center Box 102382, Durham, NC 27710, USA. Tel.: 919684 3401; fax: 919681 7950;

e-mail: courtney.thornburg@dm.duke.edu

Accepted after revision 9 January 2012 maintain factor levels above $1 \%$ to ensure effective bleed prevention. The primary goal of prophylaxis in patients with severe haemophilia is to convert the bleeding phenotype from severe to moderate and to prevent the development of chronic arthropathy $[2,3]$. Prior studies, including the randomized, controlled, Joint Outcomes Study (JOS) and the Italian randomized clinical trial of prophylaxis in children with haemophilia (the ESPRIT study) have demonstrated prophylaxis decreases joint bleeds and chronic arthropathy [4-9]. However, effectiveness of prophylaxis depends on treaters prescribing prophylaxis and patients adhering to the prescribed regimen. Prior to the publication of the JOS, we found that $86 \%$ of physicians reported that they prescribe prophylaxis on a routine basis. At the same time, only $54 \%$ of physicians reported that $76-100 \%$ of patients administer at least $80 \%$ of the 
recommended infusions [10]. The aim of this study was to determine if prescription of prophylaxis for children with haemophilia and perceptions of adherence to prophylaxis have changed since publication of the JOS.

\section{Materials and methods}

We developed a questionnaire, in electronic and written formats, based on literature review and expert opinion. The questionnaire was designed by two paediatric (SC and $\mathrm{CT}$ ) and one adult (CL) haemophilia treatment centre (HTC) physicians and two HTC nurses (JM and $\mathrm{SZ}$ ). The goal was to determine current prophylaxis practice and perceptions of adherence to prophylaxis. Excellent adherence was defined as giving $\geq 80 \%$ of prescribed infusions; this definition is commonly used in clinical trials to define adherence [11-14]. Prophylaxis was defined as long-term continuous (at least 46 weeks per year) treatment with factor. Primary prophylaxis was defined as initiated prior to the development of a target joint and secondary prophylaxis was defined as initiated after development of a target joint. The questionnaire contained 21 multiple choice questions, six Likert-scale questions, and one open-ended question (see Data S1). The questionnaire took on average $20 \mathrm{~min}$ to complete. The survey was administered to health professionals at HTCs. The Centers for Disease Control and Prevention (CDC) HTC staff database (available at https://www2a.cdc.gov/ncbddd/htcweb) [15] was used to identify physicians, physician extenders, and nurses who care for children with haemophilia. Respondents were expected to self-report based on practice at their HTC. The questionnaire was sent in hardcopy by mail and in electronic format with a link to the questionnaire in Survey Monkey. If multiple questionnaires were completed at a site, only the first questionnaire received was included.

\section{Statistical analysis}

Descriptive statistics were performed. Pearson chisquared tests and Wilcoxon tests were done to compare responses between the current respondents and respondents of the prior survey published by Thornburg [10] and to compare responses between those who reported that their prescription of prophylaxis has increased since publication of the JOS and those who reported that their prescription stayed the same.

\section{Results}

Fifty-six responses were received from health professionals at HTCs who provide care to children with haemophilia. The response rate was 56 of $128(44 \%)$ of the targeted HTCs. There were occasional missing data and denominators are provided for each response.

\section{Demographics of respondents}

Characteristics of the respondents are shown in Table 1.

\section{Prescription of prophylaxis}

Forty-eight of $55(87 \%)$ respondents reported that they prescribe prophylaxis on a regular basis compared with $86 \%(51 / 59)$ in the prior study $(P=0.91)$. Responses did not vary by role at HTC, years of experience, or size of program. Responses did vary based on or whether or not the respondent cares for both adult and paediatric patients with haemophilia. A larger portion of respondents who indicated they do not prescribe prophylaxis on a routine basis were at HTCs that primarily take care of children compared with the proportion who indicated that they do prescribe prophylaxis on a routine basis $(P=0.04)$. Health professionals were asked to report their primary reason for prescription of prophylaxis for the following age ranges: 12-23 months, $2-5$ years, $6-12$ years and $13-18$ years. The frequencies of primary and secondary prophylaxis by age were the following: age 12-23 months (primary 45/54, 83\%; secondary $9 / 54,17 \%$ ); age $2-5$ years (primary $40 / 54$, $74 \%$; secondary $14 / 54,26 \%$ ); $6-12$ years (primary $24 /$ 53, $45 \%$; secondary $29 / 53,55 \%)$; and $13-18$ years (primary 16/65, 29\%; secondary 40/56, 71\%). The frequency of prophylaxis prescription by age and type of prophylaxis (primary vs. secondary) is shown in Fig. 1 (panel a and b). Respondents were asked about factors which motivate prescription of prophylaxis,

Table 1. Characteristics of haemophilia care providers completing survey.

Survey responses, $N(\%)$

\begin{tabular}{lc}
\hline & Survey responses, $N(\%)$ \\
\hline Role at haemophilia treatment centre & \\
Physician & $36 / 56(64)$ \\
Nurse practitioner & $6 / 56(11)$ \\
Nurse & $14 / 56(25)$ \\
Experience (years) & \\
$0-5$ & $3 / 56(5)$ \\
$6-10$ & $6 / 56(11)$ \\
$11-20$ & $27 / 56(48)$ \\
$21-40$ & $19 / 56(34)$ \\
$>40$ & $1 / 56(2)$ \\
Region & \\
I & $5 / 56(9)$ \\
II & $5 / 56(9)$ \\
III & $5 / 56(9)$ \\
IV North & $3 / 56(5)$ \\
IV South & $4 / 56(7)$ \\
V West & $5 / 56(9)$ \\
V East & $10 / 56(18)$ \\
VI & $3 / 56(5)$ \\
VII & $3 / 56(5)$ \\
VIII & $4 / 56(7)$ \\
IX & $7 / 56(12)$ \\
X & $2 / 56(4)$ \\
Children treated at haemophilia treatment centre $(N)$ & \\
1-10 & $2 / 56(4)$ \\
$11-50$ & $11 / 56(20)$ \\
$51-100$ & $22 / 56(39)$ \\
$>100$ & $21 / 56(38)$ \\
\hline
\end{tabular}




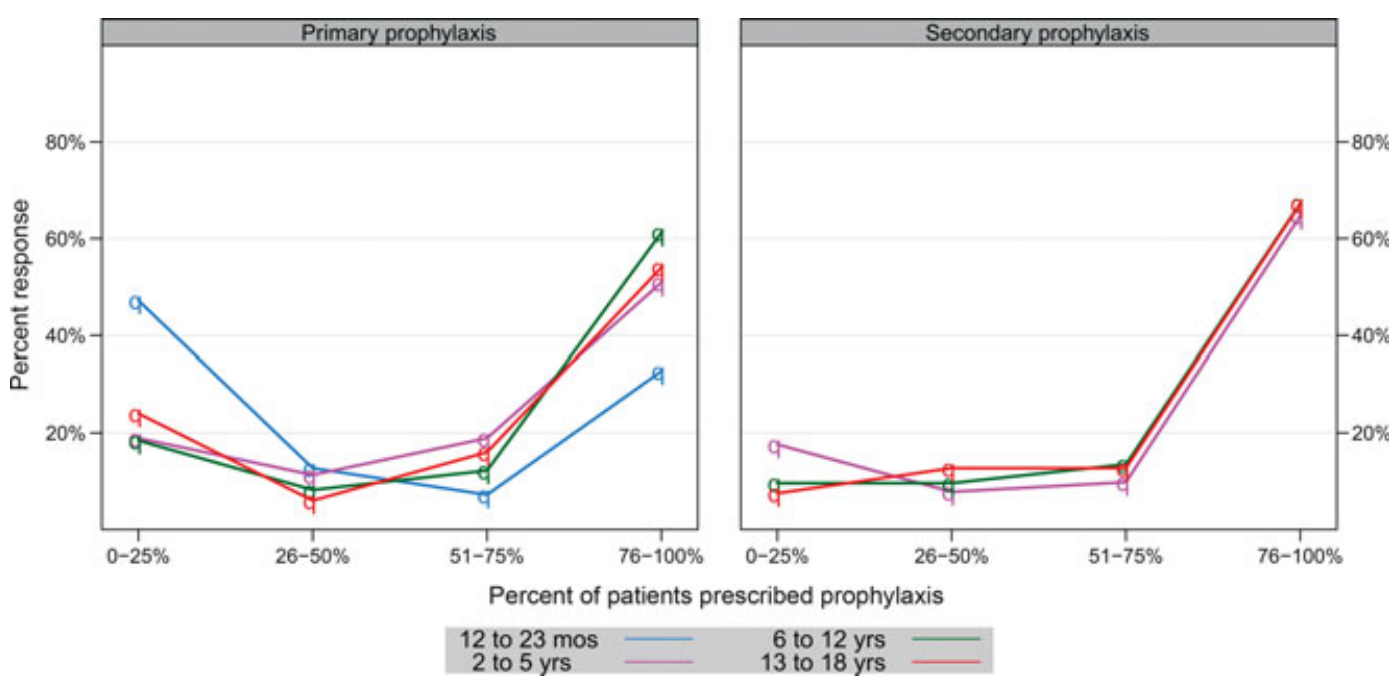

Fig. 1. Haemophilia care provided reported prescription of prophylaxis by age group. Survey respondents were asked for what percentage of their patients they prescribe primary prophylaxis (panel a) and secondary prophylaxis (panel b). Response categories included $0-25 \%, 26-50 \%, 51-75 \%$ and $76-100 \%$. Respondents were asked to provide a response for the following age groups: 12-23 months (for primary prophylaxis only), 2-5 years, 6-12 years and 13-18 years. Percentage of respondents for each category is shown on the y-axis.

factors which impact the prescription of prophylaxis, and factors which may limit the prescription of prophylaxis. These data are presented in Table 2.

\section{Impact of the joint outcomes study on the prescription of prophylaxis}

Hundred per cent of respondents strongly agreed or agreed with the conclusions of the JOS (Table 3). Fiftyfive per cent (30/55) reported the JOS increased prescription of prophylaxis, while $45 \% \quad(25 / 55)$ reported that the JOS did not impact their prescription of prophylaxis. Increase in prescription of prophylaxis since the JOS was more frequently reported by nurse practitioners and nurses compared with physicians $(P=0.03)$, providers with fewer years of experience $(P=0.01)$, and those with a smaller number of children followed at the HTC $(P=0.04)$. Of the 30 respondents who reported that the JOS increased the prescription of prophylaxis, $21 / 30(70 \%)$ reported they are more convinced that prophylaxis improves joint outcomes; $25 / 30(83.3 \%)$ reported they are able to convince more patients that prophylaxis is worthwhile; $14 / 30$ (46.7\%) report the results of the study improve adherence with prophylaxis; and 18/30 (60\%) report that insurance companies are more likely to cover prophylaxis. Of the 25 who reported the JOS did not increase their prescription of prophylaxis, 24/25 (96\%) reported they already prescribe prophylaxis for the majority of their patients and $1 / 25(4 \%)$ reported there are too many barriers to prophylaxis.

Among all of the respondents, 22/55 (40\%) reported that the Canadian study of tailored prophylaxis [16] has increased their prescription of prophylaxis and 10/55 $(18.2 \%)$ reported that the German study of early prophylaxis to prevent inhibitor formation [17] has increased their prescription of prophylaxis.

\section{Adherence and outcomes assessment}

The most common measures to assess adherence included frequency of joint bleeds $(51 / 56 ; 91 \%)$, joint examination $(41 / 56 ; 73 \%)$, hand written logs $(42 / 56$; $75 \%)$ and pharmacy records $(38 / 56 ; 68 \%)$. Only one

\begin{tabular}{lcccc}
\hline & Always/Almost & Often, & Sometimes, \\
& Always, $N(\%)$ & $N(\%)$ & $N(\%)$ & $\begin{array}{c}\text { Never, } \\
N(\%)\end{array}$ \\
\hline Factors which motivate prescription & & & & \\
$\quad$ Family history of haemarthrosis & $4 / 56(7)$ & $8 / 56(14)$ & $24 / 56(43)$ & $20 / 56(36)$ \\
$\quad \begin{array}{l}\text { Personal history of haemarthrosis } \\
\text { Personal history of soft tissue bleeds }\end{array}$ & $34 / 56(61)$ & $18 / 56(7)$ & $4 / 56(7)$ & 0 \\
$\quad \begin{array}{l}\text { Family request } \\
\text { Factors which impact prescription of prophylaxis }\end{array}$ & $9 / 56(16)$ & $18 / 55(33)$ & $20 / 55(36)$ & $5 / 55(9)$ \\
$\quad$ Family history of inhibitor & $11 / 56(20)$ & $11 / 56(20)$ & $20 / 56(36)$ & $14 / 56(25)$ \\
$\quad$ Type of genetic mutation & $4 / 56(7)$ & $11 / 56(20)$ & $20 / 56(36)$ & $21 / 56(38)$ \\
Factors which decrease prescription of prophylaxis & & & & \\
$\quad$ Financial concerns & $1 / 56(2)$ & $5 / 56(9)$ & $34 / 56(61)$ & $16 / 56(29)$ \\
Lack of venous access & $6 / 56(22)$ & $9 / 56(16)$ & $31 / 56(55)$ & $10 / 56(18)$ \\
Concerns about adherence & $2 / 56(4)$ & $7 / 56(13)$ & $40 / 56(71)$ & $7 / 56(13)$ \\
\hline
\end{tabular}

Table 2. Factors which motivate physician prescription of prophylaxis. 
Table 3. Changes in prescription of prophylaxis since publication of the Joint Outcomes Study.

\begin{tabular}{|c|c|}
\hline & $N(\%)$ \\
\hline \multicolumn{2}{|l|}{ Reasons JOS increased prescription of prophylaxis $(N=30)$} \\
\hline $\begin{array}{l}\text { More convinced that prophylaxis improves joint } \\
\text { outcomes }\end{array}$ & $21 / 30(70)$ \\
\hline $\begin{array}{l}\text { Able to convince more patients that prophylaxis is } \\
\text { worthwhile }\end{array}$ & $25 / 30(83.3)$ \\
\hline Results of study improve adherence with prophylaxis & $14 / 30(46.7)$ \\
\hline $\begin{array}{l}\text { Insurance companies are more likely to cover } \\
\text { prophylaxis }\end{array}$ & $18 / 30(60)$ \\
\hline \multicolumn{2}{|c|}{ Reasons JOS did not increase prescription of prophylaxis $(N=25)$} \\
\hline Not aware of the results of the JOS & 0 \\
\hline $\begin{array}{l}\text { Already prescribe prophylaxis to the vast majority } \\
\text { of patients }\end{array}$ & $24 / 25(96)$ \\
\hline Results not convincing & 0 \\
\hline Too many barriers to prophylaxis & $1 / 25(4)$ \\
\hline \multicolumn{2}{|c|}{ Other studies which impact prescription of prophylaxis $(N=55)$} \\
\hline Canadian study of tailored prophylaxis [16] & $22 / 55(40)$ \\
\hline $\begin{array}{l}\text { German study of early prophylaxis regimen to } \\
\text { reduce inhibitors [17] }\end{array}$ & $10 / 55(18.2)$ \\
\hline
\end{tabular}

individual indicated that they utilized a haemophiliaspecific adherence measure.

Overall, 20/48 (42\%) of respondents reported 76$100 \%$ of patients administer $\geq 80 \%$ of recommended infusions compared with $32 / 59(54 \%)$ of respondents in the prior study $(P=0.55)$. Responses indicating $76-$ $100 \%$ of patients administer at least $80 \%$ of prescribed prophylaxis did not vary by role at HTC, years of experience, size of program or whether or not respondent also cares for adult patients with haemophilia. Reported adherence decreased with increasing age group (Fig. 2). Nineteen of $56(34 \%)$ respondents reported they had not prescribed prophylaxis to at least one patient within the last year due to concerns about their patient not being adherent to the prophylactic treatment ordered and 19/56 (34\%) reported they had stopped prophylaxis for at least one patient due to concerns about the patient not being adherent with their treatment within the last year.

Respondents were queried if they would prescribe prophylaxis based on four clinical scenarios. All four scenarios concerned a two-year-old boy with severe haemophilia A with a history of haematomas, two episodes of oral bleeding and one joint bleed. In scenario 1 , the child lives with his mother (a homemaker), father (university professor) and sister, and the child does not have difficulty with venous access. In scenario 2, the home situation is the same, but venous access has been difficult for on-demand infusions. In scenario 3, the child lives with his mother (employed in several part-time jobs) and three siblings, and the child does not have difficulty with venous access. In scenario 4, the child lives with his mother (employed in several part-time jobs) and three siblings, and venous access has been difficult for on-demand infusions. The percentage of respondents who would prescribe prophylaxis for similar patients was 47/55 (85\%), 44/55 (80\%), 50/55 $(91 \%)$, and $44 / 55(80 \%)$ respectively. For scenarios 2

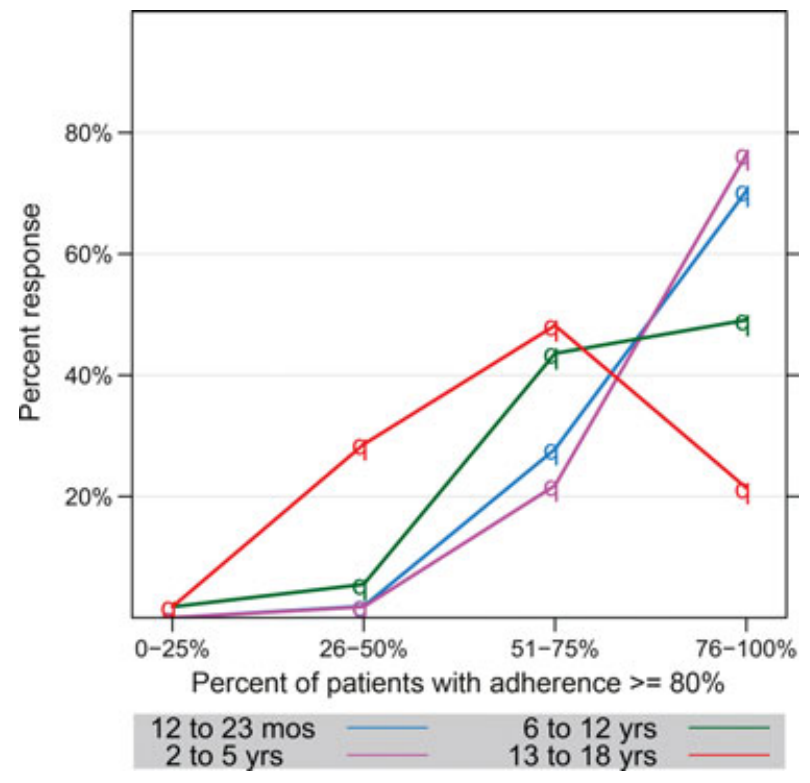

Fig. 2. Haemophilia care provider reported patient adherence to prophylaxis by age group. Survey respondents were asked what per cent of their patients administer $\geq 80 \%$ of prescribed prophylaxis. Response categories included $0-25 \%, 26-50 \%, 51-75 \%$ and $76-100 \%$. Respondents were asked to provide a response for the following age groups: 12-23 months, 2-5 years, 6-12 years and 13-18 years. Percentage of respondents for each category is shown on the $y$-axis.

and 4 , involving difficult venous access, respondents indicated that they would place a port and/or involve homecare to facilitate infusions or consider starting once-weekly regimen. For scenarios 3 and 4 one respondent reported he/she would prescribe prophylaxis 'because unexpected bleeds would be quite difficult for this family and may even lead to delays in treatment,' and another indicated 'I don't think the social situation should define prophylaxis in most cases. In this setting, bleeding would be a disaster for her jobs.'

\section{Discussion}

In our study of health professionals who prescribe prophylaxis for children with haemophilia, although the reported rate of prophylaxis was the same as with our prior study, $55 \%$ indicated that their prescription of prophylaxis increased since publication of the JOS. This is consistent with data from the CDC Universal Data Collection (UDC) program which shows that as of 2010, 53\% of patients with severe haemophilia are prescribed prophylaxis compared with $37 \%$ and $48 \%$ in 2002 and 2006 respectively [18]. Based on the survey responses, it appears the JOS has been influential on health care providers who have not previously prescribed prophylaxis for the majority of their patients, particularly nurses and nurse practitioners as well as those with fewer years of experience and those at smaller HTCs. In addition, the clinicians report that the JOS provides important information which can be used 
to educate patients about prophylaxis and can be provided to insurers.

The survey for the current study had more in depth questions regarding prescription of prophylaxis by querying practice based on age of patient and type of prophylaxis. Thirty per cent of respondents reported they prescribe primary prophylaxis to $>75 \%$ of eligible patients ages $12-23$ months compared with $\sim 50 \%$ of respondents who reported prescribing prophylaxis for $>75 \%$ of eligible patients at older ages. Respondents more often reported prescribing secondary prophylaxis to eligible patients than prescribing primary prophylaxis. These data likely reflect the fact that the primary reason for prophylaxis was a history of joint haemarthrosis. Based on the UDC study the rate of prophylaxis for children ages $2-5$ years increased from 36 to $38 \%$ between the reporting periods of 2006 to 2010 [18]. This trend may continue due to the growing evidence from the JOS [8] and ESPRIT [9] studies of the benefits of prophylaxis starting at a young age. Other motivating factors for prophylaxis included history of soft tissue bleeds, family request and family history of inhibitor. Of interest, $20 \%$ of respondents indicated that they always or almost always consider family history of inhibitor as a reason for primary prophylaxis; $75 \%$ consider it at least sometimes. Sixty-two per cent indicated they at least sometimes consider the type of genetic mutation as a reason for primary prophylaxis, indicating that clinicians are starting to consider riskbased approaches to prophylaxis prescription. In addition, $40 \%$ reported the Canadian study of tailored prophylaxis [16] has increased their prescription of prophylaxis and $18 \%$ reported the German study of early prophylaxis to prevent inhibitor formation [17] has increased their prescription of prophylaxis reflecting a tailored approach to prophylaxis. Other factors which contribute to decisions to start prophylaxis include venous access and impact of unpredictable bleeds on the child and family. Central venous catheters, nursing services and graduated prophylaxis strategy can help to facilitate prophylaxis.

\section{Barriers}

As the reported rate of prescription of prophylaxis remains high and may be increasing, prescription of prophylaxis is not universal. Only $2 \%$ of respondents indicated that financial concerns are always or almost always a limiting factor. On the other hand, only $29 \%$ per cent of respondents said that financial concerns were never an issue. Lack of access was also reported as a substantial limitation.

\section{Adherence}

Adherence is also a barrier to implementation of prophylaxis. Clinicians primarily use joint bleeds/exam- ination, hand written logs and pharmacy records to assess adherence. Although there is a newly validated measure of adherence with prophylaxis (VERITAS-Pro) [19] this measure has not been widely adopted in clinical practice. In response to a survey of Canadian haematologists and nurses, $12.5 \%$ of physicians and $17.6 \%$ of nurses indicated that they do not assess adherence [20]. Based on the adherence measures, only $42 \%$ of respondents reported that $>75 \%$ of their patients have excellent adherence ( $\geq 80 \%$ of prescribed doses) with prophylaxis. Strikingly, $\sim 70 \%$ of respondents reported that $>75 \%$ of their patients aged 1-5 years have excellent adherence compared with $22 \%$ of respondents who reported that $>75 \%$ of their adolescent patients have excellent adherence. These data are similar to those of Chan $e t$ al. where $50 \%$ of physicians in Canada indicated children were $80-100 \%$ adherent and $19 \%$ indicated adolescents were $80-100 \%$ adherent [20]. Adolescence and transition to adulthood may be a significant barrier to adherence with prophylaxis and clinicians have identified poor adherence by adolescents as a reason not to prescribe prophylaxis to that group. Up to two-thirds of young adults will experiment with stopping or reducing doses of prophylaxis when they take responsibility for selfinfusion [21], because they do not think they need prophylaxis [22].

Perceptions of adherence influence prescription of prophylaxis [10]. Richards et al. conducted a survey of 21 haemophilia doctors, throughout Europe, to study practice and opinions regarding prophylaxis in 5000 patients aged 16-24 and >50 years [23]. Eighteen of 19 doctors indicated that they would consider modifying prophylaxis in a median of $70 \%$ of adolescent/young adult patients, typically ages 16-20 years. Recommended changes included reducing the frequency of dosing, reducing the dose per infusion and changing to on-demand with option to restart in the future. This is in comparison to no doctors recommending similar changes in children $<16$ years. One of the top three reasons for these recommendations was concern about poor adherence. Similarly, Walsh and Valentino recently published a survey of US HTCs regarding factor VIII prophylaxis for adult patients with severe haemophilia A [24]. In this survey, the most important reason for not recommending prophylaxis to adult patients was concern that adults would not adhere to prophylaxis [24]. Clinicians responding to our questionnaire also indicated they may not prescribe prophylaxis or may stop prophylaxis due to concerns that their patient is not adhering to the regimen.

It is critical to address the issue of non-adherence, particularly in adolescents, as this may lead to improved outcomes. There is not a gold standard for improving adherence for medication. The need for strategies to improve medication and treatment adherence is not unique to haemophilia. Improving adherence requires a multi-faceted, tailored approach $[25,26]$. The exact 
approach depends on specific patient-related barriers to adherence. For example, if knowledge is a key barrier, strategies to improve education can be developed. Education topics should include the benefits and risks of prophylaxis including data from research studies as well as education on all of the steps required for successful administration. Education should be tailored to the health literacy of the audience and should include clear and concise information in spoken, electronic and written media. Families should be asked to explain what they know about the recommendations and demonstrate required medication administration skills. Education should continue throughout the course of prophylaxis and directed toward to the child/adolescent when appropriate. If families have difficulty remembering to order factor or to administer factor, more frequent follow-ups at the HTCs or through phone calls may be useful. Providing feedback on response can reinforce the rationale for prophylaxis; HTCs may work with families to identify the best methods by which to integrate prophylaxis into daily life. If venous access is a barrier, HTCs may elect to start prophylaxis once weekly while parents learn to infuse their children. In fact, in a recent survey by Ragni et al., greater than $30 \%$ of US HTCs indicate they initiate prophylaxis with a once weekly schedule [27]. If peripheral venous infusion is not successful, placement of a central venous catheter may facilitate prophylaxis. In the same study, $30 \%$ indicated $100 \%$ of patients require central venous catheters for prophylaxis.

The primary limitation of this study was that the data were self-reported rather than abstracted from individual site chart review. However, years and depth of experience amongst respondents supports the validity of the study. The study is also limited as we did not design the study to capture patient-reported reasons for decreased adherence.

\section{Conclusion}

Prophylaxis is an efficacious method for primary and secondary prevention of joint haemarthrosis. Ongoing research studies will lead to risk-based, tailored prophylaxis strategies. A limiting factor is adherence with prophylaxis. Strategies to improve adherence may increase the likelihood of physician prescription of prophylaxis and make prophylaxis easier to implement for individual patients, thereby improving the clinical outcome of children and adults with haemophilia. Multi-faceted and tailored approaches to improve adherence should be developed and tested.

\section{Acknowledgement}

We thank the nurses, physician extenders and physicians at the Haemophilia Treatment Centers for their participation in this study. This work was supported by an unrestricted research grant from Bayer to CT, and the investigators had full scientific independence of this work.

\section{Author contributions}

CT, SC, SZ, JM and CL designed the research, performed the research, analyzed the data and wrote the manuscript.

\section{Disclosures}

This work was supported by an unrestricted research grant from Bayer to Dr. Thornburg (CT), and the investigators had full scientific independence of this work. CT is on a physician advisory board for CSL Behring and has received speaker fees from Baxter Healthcare, Bayer and CSL Behring. SC has no competing interests to declare. SZ has acted as a paid consultant to Bayer, Baxter Healthcare, NovoNordisk, Pfizer and Grifols. JM has acted as a paid consultant to Baxter Healthcare and NovoNordisk, is on nurse advisory boards for Baxter Healthcare and CSL Behring, and is on speakers' bureaus for Bayer and NovoNordisk. CL has received research funding from Baxter Healthcare, CSL Behring, Grifols and NovoNordisk, has received speaker fees from Baxter Healthcare and CSLH Behring, and has served on advisory boards for Baxter Healthcare and CSL Behring.

\section{References}

1 Butler RB, McClure W, Wulff K. Practice patterns in haemophilia A therapy - a survey of treatment centres in the United States. Haemophilia 2003; 9: 549-54.

2 Kisker CT, Eisberg A, Schwartz B. Prophylaxis in factor IX deficiency product and patient variation. Haemophilia 2003; 9: 279-84.

3 Nilsson I, Berntorp E, Lofqvist T, Pettersson H. Twenty-five years' experience of prophylactic treatment in severe haemophilia A and B. J Int Med 1992; 232: 25-32.

4 Panicker J, Warrier I, Thomas R, Lusher JM. The overall effectiveness of prophylaxis in severe haemophilia. Haemophilia 2003; 9: 272-8.

5 Fischer K, van der Bom JG, Molho P et al. Prophylactic versus on-demand treatment strategies for severe haemophilia: a comparison of costs and long-term outcome. Haemophilia 2002; 8: 745-52.
6 van den Berg HM, Fischer K, van der Bom JG. Comparing outcomes of different treatment regimens for severe haemophilia. Haemophilia 2003; 9(Suppl 1): 27-31.

7 Fischer K, Van Den Berg M. Prophylaxis for severe haemophilia: clinical and economical issues. Haemophilia 2003; 9: 376-81.

8 Manco-Johnson MJ, Abshire TC, Shapiro AD et al. Prophylaxis versus episodic treatment to prevent joint disease in boys with severe hemophilia. N Engl J Med 2007; 357: 535-44.

9 Gringeri A, Lundin B, Mackensen SV, Mantovani L, Mannucci PM. A randomized clinical trial of prophylaxis in children with hemophilia A (the ESPRIT Study). J Thromb Haemost 2011; 9: 700-10.

10 Thornburg CD. Physicians' perceptions of adherence to prophylactic clotting factor infusions. Haemophilia 2008; 14: 25-9.

11 Bosworth H. Medication treatment adherence. In: Bosworth H, Oddone E, Weinberger $\mathrm{M}$ eds. Patient Treatment Adherence: Concepts, Interventions, and Measurement.
Mahweh: Lawrence Erlbaum Associates, Publishers, 2006: 158 .

12 Haynes RB, Sackett DL, Gibson ES et al. Improvement of medication compliance in uncontrolled hypertension. Lancet 1976; 1: 1265-8.

13 Psaty BM, Koepsell TD, Wagner EH, LoGerfo JP, Inui TS. The relative risk of incident coronary heart disease associated with recently stopping the use of beta-blockers. JAMA 1990; 263: 1653-7.

14 Osterberg L, Blaschke T. Adherence to medication. N Engl J Med 2005; 353: 48797

15 Centers for Disease Control and Prevention (CDC) HTC staff database. Available at http://www2a.cdc.gov/ncbddd/htcweb/udc_ report/udc_report.asp. Accessed 2010.

16 Feldman BM, Pai M, Rivard GE et al. Tailored prophylaxis in severe hemophilia A: interim results from the first 5 years of the Canadian Hemophilia Primary Prophylaxis Study. J Thromb Haemost 2006; 4: 1228-36. 
17 Kurnik K, Bidlingmaier C, Engl W, Chehadeh H, Reipert B, Auerswald G. New early prophylaxis regimen that avoids immunological danger signals can reduce FVIII inhibitor development. Haemophilia 2010; 16: 256-62.

18 Universal Data Collection Project. Available at https://www2a.cdc.gov/ncbddd/htcweb/ Main.asp. Accessed 2002, 2006 and 2010.

19 Duncan N, Kronenberger W, Roberson C, Shapiro A. VERITAS-Pro: a new measure of adherence to prophylactic regimens in haemophilia. Haemophilia 2010; 16: 24755.

20 Chan AK, Decker K, Warner M. Adherence with haemophilia treatments: a survey of haemophilia healthcare professionals in Canada. Haemophilia 2011; 17: 816-7.

21 Fischer K, Valentino L, Ljung R, Blanchette V. Prophylaxis for severe haemophilia: clinical challenges in the absence as well as in the presence of inhibitors. Haemophilia 2008; 14(Suppl 3): 196-201.
22 Lindvall K, Colstrup L, Wollter IM et al. Compliance with treatment and understanding of own disease in patients with severe and moderate haemophilia. Haemophilia 2006; 12: 47-51.

23 Richards M, Altisent C, Batorova A et al. Should prophylaxis be used in adolescent and adult patients with severe haemophilia? An European survey of practice and outcome data. Haemophilia 2007; 13: 473-9.

24 Walsh CE, Valentino LA. Factor VIII prophylaxis for adult patients with severe haemophilia A: results of a US survey of attitudes and practices. Haemophilia 2009; 15: 1014-21.

25 Bosworth HB, Granger BB, Mendys P et al. Medication adherence: a call for action. Am Heart J 2011; 162: 412-24.

26 du Treil S, Rice J, Leissinger CA. Quantifying adherence to treatment and its relationship to quality of life in a well-characterized haemophilia population. Haemophilia 2007; 13: 493-501.

27 Ragni MV, Fogarty PJ, Josephson NC, Neff AT, Raffini LJ, Kessler CM. Survey of current prophylaxis practices and bleeding characteristics of children with severe haemophilia A in US haemophilia treatment centres. Haemophilia 2012; 18: 63-8.

\section{Supporting Information}

Additional Supporting Information may be found in the online version of this article:

Data S1. Prophylaxis Survey 2010.

Please note: Wiley-Blackwell are not responsible for the content or functionality of any supporting materials supplied by the authors. Any queries (other than missing material) should be directed to the corresponding author for the article. 